\title{
Discours
}

Revue de linguistique, psycholinguistique et

informatique. A journal of linguistics, psycholinguistics

and computational linguistics

$8 \mid 2011$

Approches fonctionnelles de la structuration des textes

\section{Les structures argumentatives de la locution adverbiale polyvalente jusqu'à un certain point}

\section{Constanze Armbrecht}

\section{OpenEdition}

Journals

Édition électronique

URL : http://journals.openedition.org/discours/8451

DOI : $10.4000 /$ discours.8451

ISSN : 1963-1723

Éditeur :

Laboratoire LATTICE, Presses universitaires de Caen

\section{Référence électronique}

Constanze Armbrecht, « Les structures argumentatives de la locution adverbiale polyvalente jusqu'à un certain point », Discours [En ligne], 8| 2011, mis en ligne le 11 juillet 2011, consulté le 19 avril 2019. URL : http://journals.openedition.org/discours/8451; DOI : 10.4000/discours.8451

\section{c) $(1) \Theta$}

Discours est mis à disposition selon les termes de la licence Creative Commons Attribution - Pas d'Utilisation Commerciale - Pas de Modification 4.0 International. 

Revue de linguistique, psycholinguistique et informatique

\section{Les structures argumentatives de la locution adverbiale polyvalente jusqu'à un certain point}

Constanze Armbrecht

EA 1339 LiLPa

Université de Strasbourg

Constanze Armbrecht, «Les structures argumentatives de la locution adverbiale polyvalente jusqu'à un certain point», Discours [En ligne], 8 | 2011, mis en ligne le 11 juillet 2011.

URL : http://discours.revues.org/8451. Consulté le 11 juillet 2011.

Titre du numéro: Approches fonctionnelles de la structuration des textes

Éditeurs scientifiques: Laure Sarda, Shirley Carter Thomas et Benjamin Fagard

Coordination: Lydia-Mai Ho-Dac et Catherine Bolly 



\title{
Les structures argumentatives de la locution adverbiale polyvalente jusqu'à un certain point
}

Constanze Armbrecht

EA 1339 LiLPa

Université de Strasbourg

\begin{abstract}
Dans cet article nous esquissons les structures argumentatives possibles activées par l'emploi de la locution adverbiale polyfonctionnelle - quantitative et modale - jusqu'à un certain point (JCP) sur un corpus de 287 occurrences tirées du Monde. Nous montrerons quels avantages le locuteur peut retirer de l'emploi de la locution pour structurer son argumentation. Jusqu'à un certain point permet, en effet, une forme d'argumentation apparentée aux emplois du mais dit d'argumentation, connecteur auquel JCP s'associe souvent. Alors que les valeurs modales ne permettent pas à $J C P$ de construire des cadres de discours, sa portée sémantique peut organiser le discours au-delà de la phrase. Celui-ci est ainsi présenté comme argumentant en faveur de (emploi plus rare) ou contre (emploi le plus fréquent) les conclusions à tirer des propos modifiés par la locution.
\end{abstract}

Mots clés: adverbial, cadre de discours, modalité, portée sémantique, quantification, connecteur, argumentation

This paper sketches out possible argumentation structures that are activated by the use of the adverbial jusqu'à un certain point as found in a corpus of examples taken from Le Monde. It is in fact a multifunctional adverbial with both quantificational and modal properties. We show what benefit a writer may gain in using the adverbial in order to structure his or her argumentation. Jusqu'à un certain point allows one to argue in a way similar to that when using the so-called mais d'argumentation, and it is easily combined with this connector. While its modal properties will not allow it to form frames for discourse, its semantic scope can organize discourse beyond the sentence. This discourse is thus presented as either arguing in favor of (rarely) or in opposition to (most frequently) the conclusions that can be drawn from the proposition modified by the adverbial.

Keywords: adverbial, discourse frame, modality, semantic scope, quantification, connector, argumentation

\section{Introduction}

De nombreuses études explorent depuis des années la structuration du discours en mettant en évidence, entre autres, l'effet structurant de certains adverbes dits de phrase. Dans cette perspective, nous avons choisi de travailler sur la locution jusqu'à un certain point (JCP) pour deux raisons: premièrement, elle montre un fonctionnement syntaxique et sémantique hybride entre adverbial exprimant la quantification $v s$ la modalité qui complexifie ses emplois. Puis, la définition de JCP proposée par le Dictionnaire des locutions et expressions (DLE) ne rend pas complètement compte de son sens: la paraphrase «en partie, de façon partielle» $(D L E)$ n'explicite pas l'idée 
que certains critères nécessaires pour affirmer entièrement un propos ne sont pas satisfaits. C'est cette notion d'insatisfaction pourtant, qui porte en elle le potentiel d'une orientation argumentative. Elle peut annoncer un volet de discours qui mettra en évidence ce pourquoi le propos ne peut être totalement vrai. La portée sémantique de $J C P$, alliant quantité et modalité, ainsi que son caractère scalaire, permettent un enchaînement particulier du discours dont nous relèverons les affinités avec le connecteur mais.

L'intérêt de la présente étude est de mettre en lumière un type d'item de discours peu étudié qui touche différents domaines (quantification, modalisation, argumentation) et fonctions (adverbial, connecteur), ainsi que d'esquisser les manières dont une telle forme hybride contribue à la structuration du discours. Notre analyse s'appuie sur un corpus constitué des occurrences complètes de la locution dans les numéros du Monde de 1980 à 2006 sur CD-ROM.

Nous présenterons d'abord la polyfonctionnalité de la locution adverbiale $J C P$ et son caractère polarisant (polarity item). Les exemples du corpus ${ }^{1}$ utilisés dans cette partie descriptive seront complétés par des exemples construits et des occurrences tirées de Frantext. Ensuite, nous proposerons un fonctionnement argumentatif dans la lignée d'Anscombre et Ducrot (1983; Ducrot, 1993) pour JCP, dont nous dégageons deux types d'emploi en discours. Nous présenterons, enfin, les résultats de l'étude du corpus et les types de structure argumentative que nous avons pu y relever. L'analyse s'intéressera en particulier à $J C P$ en position finale où la locution développe toute sa force argumentative ${ }^{2}$.

\section{Caractéristiques syntaxiques et sémantiques d'un hybride : jusqu'à un certain point entre quantification et modalisation}

\subsection{Emplois endophrastique et exophrastique de $J C P$}

Comme bon nombre d'adverbes, $J C P$ peut fonctionner à deux niveaux discursifs. Il peut contribuer au sens référentiel des phrases, donc désigner des éléments externes au discours par rapport auxquels l'adverbe dit quelque chose. Il peut aussi refléter la conscience du locuteur de l'évolution de ses propos. En d'autres termes, JCP peut être endophrastique ou exophrastique (terminologie de Guimier, $1996^{3}$ ). Cette

1. Les exemples du Monde sont indiqués (M).

2. Nous remercions chaleureusement C. Schnedecker, B. Fagard, V. Lenepveu et A. Le Draoulec pour leurs relectures, conseils et commentaires instructifs.

3. Tandis que l'incidence est définie comme la référence syntaxique à un support, niveau auquel on peut distinguer entre adverbes intra-prédicatifs et adverbes extra-prédicatifs, la portée concerne la «référence sémantique, c'est-à-dire l'élément à propos duquel l'adverbe dit préférentiellement quelque chose» (Guimier, I996: 4). À ce niveau, les adverbes endophrastiques «affectent le contenu même de l'élément sur lequel ils portent et, ce faisant, participent à la construction du sens référentiel de la phrase» (Guimier, I996: 6). Les adverbes exophrastiques ne participent pas à la construction du sens référentiel, «mais 
différence est mise au clair par les paires dans [I]. Endophrastique, JCP ne peut être détaché en tête de phrase sans que son sens en soit modifié. Mais il peut être repris par un adverbe d'intensité comme davantage.

[I] Je l'aime jusqu'à un certain point.

\# Jusqu'à un certain point, je l'aime.

Je l'aime jusqu'à un certain point, non davantage.

* Jusqu'à un certain point, je l'aime, non davantage.

Exophrastique, $J C P$ peut s'antéposer à une phrase négative:

[2] Jusqu'à un certain point, je ne l'aime pas.

Il se comporte comme un adverbe modal, modulant le degré d'assertion du contenu entier. Il se paraphrase alors par «à certains égards», «d'un certain point de vue» ${ }^{4}$ :

[3] Jusqu'à un certain point, l'Église fut atteinte elle aussi par le refus des hiérarchies. $\rightarrow$ D'un certain point de vue / À certains égards, l'Église en fut atteinte aussi.

$(\mathrm{M})$

Deux types de lecture modale sont possibles: JCP peut fonctionner comme shield (terme de Prince, Frader et Bosk, 1982, cité dans Mihatsch, 20ıO), en d'autres termes comme enclosure (terme de Kleiber et Riegel, 1978) à portée large. Il touche au statut vériconditionnel de la phrase entière et protège le locuteur dont les propos ont moins de force car l'assertion en est affaiblie, par exemple dans:

[4] Inexplicable aussi restera toujours, pour l'essentiel, quoi qu'on dise, l'art du comédien, celui de cette immense actrice qu'est Maria Casarès (Hécube). [...] Et jusqu’à un certain point il en va de même des acteurs qui entourent Maria Casarès [...].

(M)

Lorsqu'il porte sur un seul constituant, $J C P$ forme une enclosure à portée étroite. L'adverbial est, en d'autres termes, utilisé en tant que bedge, précisément avec la fonction d'approximator (Prince, Frader et Bosk, 1982) qui marque la relative adéquation d'un terme utilisé à la situation décrite. On peut paraphraser par «qu'on peut ou ne peut pas appeler», voir l'exemple [s]:

[5] C'est ainsi que je devins l'hôte et, jusqu'à un certain point, l'ami des S. [...].

$\rightarrow$ Je devins ce qu'on peut ou ne peut pas appeler ami.

(A. Billy, Intrö̈bo, 1939)

représentent des traces de l'intervention du locuteur, qui commente tout ou partie de son énoncé ou de l'acte qui le produit» (ibid.).

4. Il est clair que les paraphrases ne marquent jamais l'équivalence exacte. Ainsi, dans un travail en cours, nous cherchons à dégager des contextes différenciateurs entre JCP et les paraphrases proposées ici. 
Endophrastique, $J C P$ tombe sous la négation, [6], ou l'interrogation, [7], et peut être repris par un adverbe quantifieur intensif, [8], ou de complétude ${ }^{5}$, [9], selon le cas ${ }^{6}$ :

[6] Ceux qui décident de dialoguer entre eux ne se comprennent l'un l'autre - et ne se comprennent eux-mêmes - que jusqu'à un certain point.

(M)

[7] En résumé, faut-il limiter les interventions de l'État-providence jusqu’à un certain point?

(M)

[8] J'aime cette fille jusqu'à un certain point, non davantage [...].

(H. de Montherlant, Le Démon du bien, 1937)

[9] Je suis d'accord jusqu'à un certain point, pas complètement.

En dépendance du prédicat et du cotexte, $J C P$ exprime le plus souvent l'idée d'un seuil critique au-delà duquel le prédicat n'est plus valable ou dont le dépassement déclenche un effet contraire. L'interprétation de cette notion de délimitation dépend toujours du contexte ${ }^{7}$ précis dans lequel s'insère $J C P$. Par exemple:

[ro] Je peux débattre d'un sujet jusqu'à un certain point, mais après... Marie dit toujours que nous, les Américains, sommes des gens plus prêts à la confrontation.

$\approx \mathrm{Il} \mathrm{y}$ a des limites à ma disponibilité de discuter, si l'on insiste trop sur certains sujets avec moi, je cesse d'être coopératif.

(M)

On voit comment cette idée de limite est activée et actualisée métaphoriquement ${ }^{8}$, ce qui s'exprime par des formes assimilables à des anaphores spatio-temporelles (qui sont des éléments endophrastiques) renvoyant à point, comme encore dans :

[II] Mais cette affection, pourtant véritable, va jusqu'à un certain point, non au-delà. (H. de Montherlant, Pitié pour les femmes, 1936)

[I2] Il faut penser qu'il [le pouvoir] peut étendre les bases sur lesquelles il repose jusqu’à un certain point seulement, après quoi il se heurte comme à un mur infranchissable. (S. Weil, Euvres, 1929-1943, t. III)

5. Terme de Molinier et Levrier, 2000 .

6. Nous proposons une discussion approfondie de la quantification adverbiale dans notre travail de recherche en cours. Sur la question, voir par exemple Caudal et Nicolas, 2005; Lenepveu, 2009.

7. Nous entendons par là et le cotexte, i. e. l'entourage grammatico-lexical, et le contexte, i. e. les connaissances du monde saillantes dans l'interprétation d'un morceau de discours.

8. Si on accepte de considérer la transposition du nom point à des domaines abstraits au-delà de l'espacetemps, comme une métaphorisation. 
[13] Jusqu'à un certain point il y a un cap. Puis tout se dérobe à la façon d'un vol [...]. (Internet)

$J C P$ se démarque également comme endophrastique lorsqu'il est combiné avec un adverbe de modalisation. Ainsi, dans cet exemple où il apparait comme substituant pour la prophrase «Oui» et peut être paraphrasé par un adverbial quantifieur de complétude:

[I4] [C]es trois genres dramatiques sont-ils devenus des «théâtres-musées» [...] ? Jusqu’à un certain point, certainement.

$\rightarrow$ Oui, certainement.

$\rightarrow$ Jusqu'à un certain point, certainement, mais pas complètement / pas tout à fait. $(\mathrm{M})$

\section{2. $J C P$, un hybride entre quantification et modalisation}

Néanmoins, on doit reconnaître une certaine difficulté à distinguer les valeurs quantitative et modale dans la plupart des cas, voir ainsi :

[I5a] Il a raison jusqu’à un certain point.

$(D L E)$

[Isb] Il a jusqu'à un certain point raison.

[ısc] Jusqu'à un certain point, il a raison.

L'ambiguïté de portée dans des cas comme [I $[5]$ est d'autant plus difficile à lever que les tests habituels de paraphrase (cf. Molinier et Levrier, 2000; Guimier, 1996) ne peuvent s'appliquer directement à $J C P$ par manque de base adjectivale ${ }^{9}$.

En effet, la plupart des occurrences présentent des lectures mixtes quantitative et modale. Ces lectures hybrides naissent, d'une part, lorsque $J C P$ se combine avec des prédicats à arguments quantifiables, voir par exemple:

[16] Je pensais que l'avenir l'effrayait et je partageais d'ailleurs son appréhension, jusqu'à un certain point.

(M. Del Castillo, La Nuit du décret, 1981)

[16a] Je la partageais jusqu’à un certain point, pas davantage, pas entièrement, pas au-delà de quelques sujets bien précis.

[16b] À certains égards, je la partageais.

9. Nous considérons que la paraphrase par l'adjectif certain n'est pas correcte, cf. aimer jusqu'à un certain point $\neq$ avoir un certain amour pour. 
[17] Jusqu'à un certain point, elles savaient ce qu'il allait leur dire: les foules savent souvent ce qu'on va leur dire.

(A. Malraux, Antimémoires, 1976)

[r7a] Elles savaient en partie ce qu'il allait leur dire.

[ı7b] À certains égards, elles savaient ce qu'il allait leur dire.

Dans ces exemples, JCP porte sur un argument du prédicat verbal, ici les COD «appréhension» et «ce qu'il allait leur dire». Si l'on admet que ces arguments peuvent conceptuellement être constitués de «certains aspects de l'avenir qui effraient» ou d'un nombre d' «éléments formant le discours», $J C P$ peut être considéré comme quantifiant en délimitant une partie de l'appréhension ou certains des éléments du discours évoqué ${ }^{10}$.

Le sémantisme de $J C P$ incite à conceptualiser le prédicat comme un cheminement le long de faits définitoires alignés, jusqu'à un moment de «bascule» et permet ainsi d'autres lectures quantitatives. L'idée de parcours à travers des situations qui vérifient le prédicat peut être sentie comme chronologique. Dans [I8], JCP prend une valeur temporelle - via l'idée du déroulement du match - parallèle à un sens modal:

[18] Sven-Göran Eriksson avait prévu de priver de ballon les milieux de terrain et les attaquants français. Son plan avait fonctionné. Jusqu’à un certain point... Le bonheur que j’ai ressenti au moment de l'égalisation, puis du pénalty, synonyme de victoire [...]. (M)

Dans de tels cas, $J C P$ rend une activité télique en bornant le procès. Comme le note Lenepveu (2009), l'effet est quantitatif car il y a une dimension (dans [I8], le temps) sur laquelle la quantification peut porter.

Autrement, selon le prédicat, JCP peut quantifier la dimension de l'intensité, comme avec le verbe «aimer». Le cheminement est conceptualisé «en profondeur», selon les termes d'Adler et Asnes (2004), sur l'axe de l'intensité (cf. [8]) ${ }^{11}$ :

aimer peu < aimer jusqu’à un certain point < aimer beaucoup $<\ldots$

L'effet «mixte» (modal-quantifieur) des emplois quantitatif et intensif est renforcé par la double articulation des quantifieurs indéfinis: ils quantifient des

10. Nous n'aurions pas la place ici pour discuter les éléments de la quantification adverbiale qui jouent dans le calcul du sens endo- et exophrastique d'une occurrence. Nous discutons les pistes ouvertes par Lenepveu (2009) et l'idée d'arguments internes (cf. Molinier et Levrier, 2000) dans notre travail en cours. Molinier et Levrier proposent des exemples comme Max a beaucoup saigné où l'argument interne est «du sang», pour arriver à une quantification sous-jacente de beaucoup sur sang. Ce que nous appelons ici provisoirement «un argument interne associé», ce sont des éléments qu'on associe avec le sémantisme du prédicat verbal sans que celui-ci permette de le dériver comme sang de saigner.

11. La gradation que nous proposons ici ne prend pas en compte l'orientation argumentative des quantifieurs, telle qu'elle est proposée par O. Ducrot et que nous abordons dans la partie 2. 
référents, mais en lien avec une estimation subjective - peu, beaucoup, etc. "par rapport à X»- qui se situe à un niveau métalinguistique.

Comme les exemples cités le montrent, l'idée de cheminement est souvent purement métaphorique : le mouvement est «abstrait» ou «fictif» (cf. la notion de fictive motion des approches cognitives, comme celles de Lakoff et Johnson, 1980; Langacker, 1987 et Talmy, 2000). Le prédicat n'est pas une expression d'étendue ou de mouvement. Le parcours exprimé par JCP est donc un parcours «imagé» le long des faits définitoires qui vérifient le prédicat, en dehors de l'ancrage spatio-temporel à un niveau notionnel. Il s'agit donc de considérer son degré d'adéquation à la situation décrite qui est presque donnée comme un alignement de traits définitoires. De la même manière qu'une pelouse est une étendue d'espace car elle est couverte de $\mathrm{A}$ à $\mathrm{B}$ d'herbe, dans [8], l'«espace» de la notion d'amour est couvert en partie, mais pas en totalité. C'est donc de l'amour tout en ne l'étant pas. JCP est modal en ce qu'il restreint l'applicabilité du prédicat. Il a pourtant une charge quantitative endophrastique en même temps, car il se réfère à un ensemble indéfini de traits qui peuvent ensuite être explicités dans le contexte droit.

Il se dessine alors ce qu'affirme Lenepveu (2009) pour les adverbes de complétude qu'elle étudie $^{12}$ : l'emploi modal ne s'affranchit pas complètement de la valeur quantifiante (cf. [16], [17]).JCP active une notion de limite qui porte parallèlement sur des éléments référentiels (l'espace-temps, des critères définitoires du prédicat réalisés ou non) et sur l'appréhension de l'énoncé par le locuteur (il y adhère à certains mais pas à tous points de vue). Cette activation parallèle semble aussi permettre l'inverse à $J C P$, c'est-à-dire d'indiquer quasiment toujours, plus ou moins fortement selon le cas et pour les raisons que nous avons évoquées, une valeur modale.

\subsection{La position de $J C P$ dans la phrase et ses effets sur l'interprétation de l'adverbial}

Les lectures - quantitative, modale ou mixte - sont, bien sûr, influencées par la place syntaxique de $J C P$.

L'antéposition de l'adverbial à la phrase entière le désigne presque toujours nettement comme modal, ainsi :

[19] Jusqu'à un certain point, la Russie aussi comprend que pour faire progresser le processus de paix, Yasser Arafat doit être évincé.

(M)

Bien que cette position en tête de phrase soit caractéristique des adverbes exophrastiques (cf. Guimier, 1996; Molinier et Levrier, 2000), elle accepte bon nombre de circonstants endophrastiques, notamment les spatio-temporels (cf. entre

12. Complètement, entièrement, totalement, intégralement (Lenepveu, 2009). 
autres Charolles, 2003). Si on accorde à $J C P$ une (faible) valeur spatio-temporelle, comme dans $[\mathrm{I} 3]$ repris ici sous [20]:

[2o] Jusqu'à un certain point il y a un cap. Puis tout se dérobe [...].

(Internet)

on pourrait imaginer que l'antéposition en tête de phrase permette un emploi endophrastique de la locution. En réalité, on s'aperçoit que de telles constructions sont stylistiquement marquées (cf. l'exemple ci-dessus). Un véritable emploi spatio-temporel réactive, en effet, le nom point et brise le caractère figé de l'adverbial, voir ainsi:

[2I] Jusqu'à un certain point, la forêt continuait à bloquer toute lumière, et puis, soudain, une clairière parsemée de lis sauvages.

$J C P=$ jusqu’à un endroit, un moment, mais pas plus loin ou plus longtemps que...

La postposition est canoniquement celle du complément circonstanciel ${ }^{13}$. C'est la position de $J C P$ endophrastique :

[22] La femme est sa mère, Ann Brancroft, mère juive, compréhensive jusqu'à un certain point.

$\rightarrow$ Compréhensive jusqu’à un certain point, non davantage.

(M)

[23] J'aime la solitude jusqu'à un certain point.

$\rightarrow$ J'aime la solitude jusqu'à un certain point, non davantage.

(M)

Mais cette position à elle seule n'a rien de décisif: la postposition est aussi celle des adverbes modaux (cf. Molinier et Levrier, 2000: 91 sq.; Guimier, 1996: II2 sq.; ex. il viendra certainement), la position rhématique de l'adverbe (cf. Guimier, 1996: 46; ex. ils forment chaque vase manuellement) ou le lieu d'ajouts tardifs (Guimier, 1996: 17; ex. nous étions partis main dans la main, soulagés, immensément). Il reste difficile de trancher entre quantitatif (pas davantage/complètement) et modal (à certains égards) si le prédicat et le cotexte ne donnent pas d'indications qui font nettement pencher vers l'une ou l'autre interprétation, ainsi :

[24] Cela a fonctionné jusqu'à un certain point. Un des signes en a été l'apparition de la fameuse oligarchie $[\ldots]$.

$\rightarrow$ Cela a fonctionné jusqu’à un certain point mais pas à cent pour cent. / Cela a fonctionné pendant un certain temps mais pas plus longtemps / À certains égards, cela a fonctionné.

(M)

13. En diachronie, $J C P$ occupe cette place avant de s'en affranchir pour enfin occuper la position modale en tête de phrase. 
Dans d'autres cas, l'ambiguïté peut être levée par le contexte droit si celui-ci marque clairement la fermeture d'un mouvement argumentatif. La lecture est exophrastique modale lorsqu'un nouveau mouvement d'argumentation s'ouvre grâce à un marqueur d'argumentation tel que cependant, quand même, mais, comme dans:

[25] C'était convaincant, jusqu'à un certain point. L'Espagne a quand même été probablement ciblée pour son soutien à la guerre de Bush en Irak [...].

$\rightarrow$ À certains égards, c'était convaincant.

(M)

La position postverbale de $J C P$ favorise souvent une lecture exophrastique modale (bedge) plutôt que quantitative:

[26] Ils reflètent jusqu'à un certain point un antiaméricanisme qui n'est plus aujourd'hui le fait d'une gauche défunte mais du conservatisme.

$\rightarrow$ À certains égards, ils reflètent un antiaméricanisme [...].

$(\mathrm{M})$

Mais là encore, tout dépend largement du contexte.

\subsection{La valeur scalaire de $J C P$}

$J C P$ est une locution scalaire et peut être considéré comme un adverbial assertif restrictif (terme de Nøjgaard, 1995; cf. aussi Guimier, 1996) ${ }^{14}$. Ainsi, JCP a une valeur positive, assertive, et peut se substituer à la prophrase «Oui» mais non à la négative:

[27] Tout cela signifie-t-il que la détente est en vue?

Oui.

Oui, jusqu'à un certain point.

Jusqu'à un certain point.

* Non, jusqu’à un certain point.

(M)

Ces faits démontrent que lorsque JCP porte sur une proposition entière, il affirme son contenu. En même temps, il situe le constituant, prédicat ou énoncé sur lequel il opère, en bas d'une échelle de valeurs. Il peut ainsi se combiner avec des éléments minimisants comme au moins ou restrictifs comme seulement, ne... que... :

[28] L'Acte unique européen ne parle pas des cotisations sociales qui sont aussi un prélèvement obligatoire. L'homogénéisation de l'impôt, qu'implique au moins jusqu'à un certain point le marché unique, n'exige-t-elle pas qu'elles soient également prises en compte?

(M)

14. Les adverbiaux assertifs restrictifs font référence au possible ou probable et suspendent l'assertion en ce qu'ils «ne présentent pas comme acquise la validité de l'énoncé auquel ils sont incidents» (Guimier, I996: II4). 
[29] Ce propos se tient jusqu'à un certain point seulement, car l'État - on le voit bien en Corée ou à Taïwan - a un rôle important à jouer.

(M)

[30] [...] tout ce qui nous entoure n'est vrai que jusqu'à un certain point.

(A. Béguin, L'Âme romantique et le rêve: essai sur le romantisme allemand et la poésie française, 1939)

La zone opposée, en haut de l'échelle, visée par le restrictif augmentatif même (terme de Nøjgaard, 1995) est inaccessible avec JCP:

[3I] [...] on ne peut tirer ici des conséquences vérifiables que jusqu'à un certain point [...]. * [...] on peut tirer ici des conséquences vérifiables même jusqu’à un certain point.

(H. Bergson, L'Évolution créatrice, 1907)

[32] [...] un être qu'il n'aimait que jusqu'à un certain point!

* [...] un être qu'il aimait même jusqu'à un certain point.

(H. de Montherlant, Le Démon du bien, 1937)

Ce trait scalaire explique la part de négation dans $J C P$, sa prédisposition à opérer négativement sur l'élément ou l'assertion sous sa portée et à ouvrir sur un discours qui sera présenté comme s'y opposant. Nous allons le voir en détail dans la partie suivante.

\section{JCP: un opérateur argumentatif}

\subsection{Un modèle possible: les espaces mentaux}

Après les observations sur le caractère hybride de ses valeurs sémantiques, quel type de structuration - si type particulier il y a - JCP peut-il introduire dans le discours? Il est clair que la locution ne peut être envisagée comme un «topique cadratif» tel que présenté dans Charolles (2003; Charolles et Vigier, 2005). Nous avons vu que la valeur spatio-temporelle de $J C P$ en tête de phrase ${ }^{15}$ est trop faible pour en faire un cadre à charge véridictionnelle ${ }^{16}$. En effet, les adverbiaux modaux, les circonstanciels qualitatifs ainsi que les compléments de degré sont généralement exclus comme topiques (Charolles, 2003; Charolles et Vigier, 2005). L'explication est nette: «Les adverbes modaux du type forcément, nécessairement, probablement, peut-être, etc. sont des opérateurs vériconditionnels qui affectent le calcul de la vérité/fausseté du contenu de la proposition dans laquelle ils figurent. Par contre, les adverbiaux cadratifs [...]

15. Charolles (1997) montre que le fonctionnement comme topique cadratif semble réservé à la position détachée en tête de phrase.

16. Une valeur véridictionnelle «n'impose pas un certain mode de calcul de la vérité/fausseté [d'une proposition]». Elle est «purement interprétative en ce sens qu'elle demande à l'auditeur de relativiser la vérité de [la proposition] au seul univers spécifié» (Charolles, 1997:24). 
interviennent, non pas sur le contenu propositionnel de la proposition en tête de laquelle ils apparaissent, mais sur le fait qu'elle dénote, ce qui n'est possible qu'une fois que celui-ci a été construit comme tel, donc une fois que sa valeur modale a été calculée» (Charolles, 2003: 18). Comme nous l'avons vu sous I.3 (cf. [20], [21]), une telle séparation n'est pas possible avec JCP en tête de phrase sans briser son caractère figé et réactualiser point comme un nom libre.

En même temps, nous avons vu que $J C P$ ne se comporte pas souvent comme un pur modal (cf. I.3) et peut facilement lier la quantification au prédicat si celui-ci le permet sémantiquement. En effet, il faudrait une conception qui lie la part référentielle visant «la réalité» partagée des interlocuteurs et la part modale opérant sur la validité de l'assertion. Passons alors à un exemple pour étoffer la discussion et argumenter en faveur d'un fonctionnement space builder (terme de Fauconnier, 1985) de JCP. Les space builders sont de nature variable et appréhendés de manière différente selon les auteurs (voir par exemple Charolles, 1997, pour un aperçu). L'approche de Charolles utilise le concept des cadres qui regroupe différents types de marquage indiquant «que plusieurs unités doivent être traitées de la même manière relativement à un critère (plus ou moins) spécifié par ces expressions» (Charolles, 1997: 3) et référer à un «ensemble de circonstances [...] dans lesquelles la proposition peut être dite vraie» (Charolles, 1997: 5, définition de Martin, 1983, reprise par l'auteur). Ces définitions renvoient aux cadratifs véridictionnels, desquels nous avons écarté $J C P$, mais elles incluent également des adverbiaux comme en général/ en particulier (Charolles, 1997). Ces adverbes non seulement ouvrent des «univers de discours», mais y associent des «univers parents» et nous pensons retrouver cet effet avec JCP. Comme en général, qui appelle un univers parent, en particulier, JCP appelle l'idée de quelque chose qui manque pour présenter une affirmation comme «totalement vraie». Cette idée n'a pas de forme linguistique, comme le lexème en particulier qui répond à en général. Mais elle est accessible en discours et peut y être présente sous forme de propositions. Pour illustrer cette conception nous recourons au modèle de Fauconnier qui, de manière assez large, inclut parmi des space builders des attitudes discursives (espérer, penser), la négation et la modalité (il est possible que...). Sous cet angle, nous pouvons considérer $J C P$ comme un "créateur d'espaces mentaux». Soit cet extrait d'un article du Monde:

[33] La fronde des jeunes élus est-elle porteuse de renouveau? Jusqu'à un certain point. Leur agacement est réel mais ils sont partagés entre l'appartenance à un clan qui sert leur carrière (par le financement et les promotions) et les aspirations des électeurs. (M)

L'exemple ${ }^{17}$ montre comment JCP porte sur une proposition («la fronde des jeunes élus est porteuse de renouveau») dont la véracité sera soutenue par le discours qui suit («leur agacement est réel»). Dans un tel cas, $J C P$ avertit le lecteur d'ouvrir

17. Il s'agit d'une question rhétorique attribuée au lecteur ou à un autre interlocuteur fictif mis en scène par le rédacteur. La structure correspond donc à une affirmation modifiée par JCP. 
un «espace mental» qui recense tous les arguments donnés en faveur de ce qui a été asserté (EI). Ces arguments peuvent rester virtuels ou, comme dans l'exemple, apparaitre dans le cotexte droit. En même temps, $J C P$ introduit un autre espace de «totalité» $\left(\mathrm{E}_{3}\right)$, et un espace $\left(\mathrm{E}_{2}\right)$ qui représente la différence entre l'espace I (les aspects assertés) et l'espace de «totalité». Cette différence contient tout ce qui manque pour que la proposition soit totalement vraie. Dans l'exemple, cet espace de «différence» est actualisé par le propos introduit par «mais». On peut parler de ces deuxième et troisième espaces comme d'espaces parents (terminologie de Charolles), univers «qui demeurent virtuels tant qu'ils ne sont pas instanciés dans le texte» (Charolles, 1997: 7).

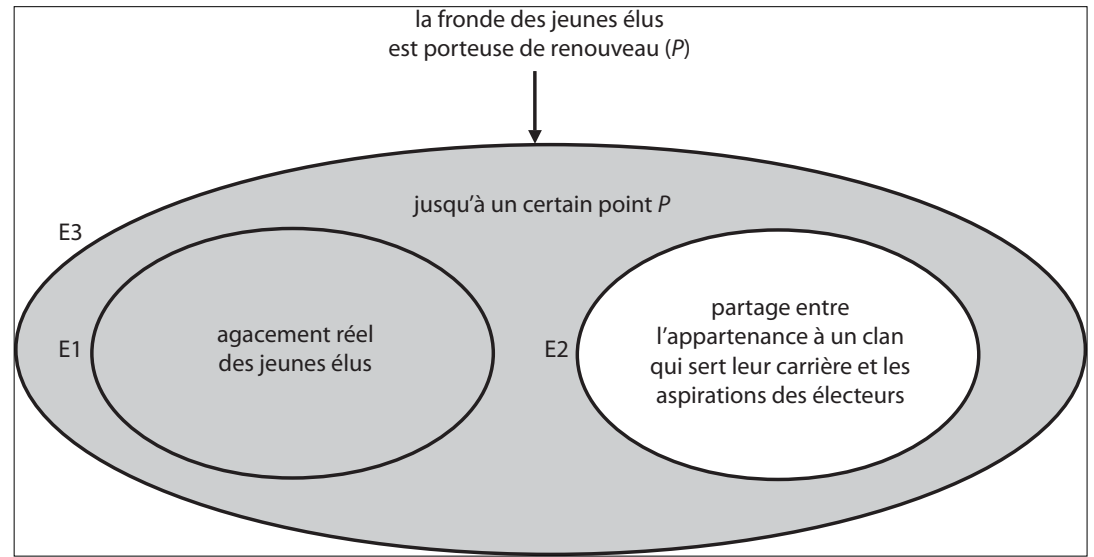

Schéma 1. Les trois espaces mentaux construits par JCP

Dans un autre cas de figure, c'est l'espace I, le côté affirmatif, qui reste virtuel, tandis que l'espace 2, où se situent les éléments manquants, la part d'insatisfaction du prédicat, est actualisé :

[34] Sans aller jusqu'à la «monarchie de la bicyclette» des pays scandinaves, la maison impériale nippone se veut plus «ouverte». Jusqu'à un certain point: lorsque Akihito visita récemment le musée des Sciences dans le parc de Ueno à Tokyo, la police fit déguerpir la foule de pauvres hères qui bivouaquent sous les arbres pour que leur présence ne choque pas l'auguste regard.

Dans un troisième exemple, ni l'espace i ni l'espace 2 ne sont actualisés. On notera pourtant que la suite «mais enfin» est seulement possible en opposition à l'espace 2 où se situe la part d'insatisfaction:

[35] Le rêve américain est toujours debout, les jeunes veulent changer le monde, les parents tiennent à se montrer larges d'esprit. Jusqu'à un certain point, d'ailleurs. Mais enfin tout va bien.

(M) 
Dans un texte, plusieurs propositions (phrases) peuvent potentiellement remplir chacun des espaces I et 2 (cf. les exemples de 2.3 et 3). On l'aura remarqué, différentes structures syntaxiques et énonciatives conditionnent la focalisation sur l'un ou l'autre de ces deux espaces. Nous les préciserons par la suite.

On voit ainsi que la portée sémantique de $J C P$ s'étend au-delà du cadre de la phrase pour avoir des effets structurants au niveau du discours. L'ouverture de ces espaces permet notamment un jeu polyphonique d'opposition lorsqu'un locuteur peut attribuer la prise en charge de l'espace i à une autre source tandis que lui-même assume l'espace 2. Dans la partie suivante, nous regarderons comment JCP fonctionne particulièrement comme opérateur d'orientation argumentative.

\subsection{Opérateur argumentatif à valeur négative: rôle particulier de $J C P$ indépendant}

Dans la «Théorie de l'argumentation dans la langue» (Anscombre et Ducrot, I983), des opérateurs comme peu, un peu, presque ou à peine ont reçu une attention particulière. L'idée essentielle est que ces opérateurs argumentatifs entrent dans la structure sémantique de la phrase pour lui conférer une orientation vers un certain type de conclusion. La conclusion ne serait pas déduite du seul contenu informationnel de la phrase (Van Eemeren et al., 1996). Par exemple:

[36] a Il y avait vingt personnes.

Donc la fête fut un succès.

Donc la fête fut un échec.

b Il y avait presque vingt personnes.

Donc la fête fut un succès.

* Donc la fête fut un échec.

c Il y avait à peine vingt personnes.

* Donc la fête fut un succès.

Donc la fête fut un échec.

(Van Eemeren et al., 1996; nous traduisons)

Les opérateurs sont des outils originaux pour «présenter un énoncé Eı (ou ensemble d'énoncés) comme destiné à en faire admettre un autre (ou un ensemble d'autres) E2» (Anscombre et Ducrot, I983: 8) au lecteur ou interlocuteur. La série a ci-dessus montre comment, en l'absence de l'opérateur, cette orientation argumentative reste ambiguë. Ainsi, la présence d'un opérateur conditionne aussi la suite du discours, comme le montrent les phrases acceptables (de a à c) ci-dessus.

$J C P$ peut endosser un tel fonctionnement d'opérateur argumentatif. Nous avons exposé le caractère scalaire de $J C P$, affirmatif restrictif, sous i.4. En effet, JCP peut mobiliser le côté «affirmatif» et le côté «négatif» séparément. Pour être introduit par mais dans un mouvement de contre-argumentation, JCP doit avoir une valeur restrictive, négative: 
[37] Nous devrions laisser jouer la loi du marché, mais jusqu'à un certain point. Qu'est-ce que le marché, si ce n'est la loi de la jungle, de la nature, alors que la civilisation, c'est la lutte contre la nature.

* Nous devrions laisser jouer la loi du marché, [mais] ø. Qu'est-ce que le marché, si ce n'est la loi de la jungle, de la nature, alors que la civilisation, c'est la lutte contre la nature.

(M)

Cependant, cet effet de négation ouvrant sur une contre-argumentation ne peut se produire que lorsque $J C P$ fonctionne comme une affirmation indépendante. Nous devons distinguer cet emploi d'un emploi intégré dans la prédication. L'indépendance est marquée par une ponctuation plus ou moins forte (virgule, point, points de suspension, deux-points, mais). Une légère modification de l'exemple précédent va le montrer:

[38a] Nous devrions laisser jouer la loi du marché, jusqu'à un certain point. Qu'est-ce que le marché, si ce n'est la loi de la jungle, de la nature, alors que la civilisation, c'est la lutte contre la nature.

[38b] * Nous devrions laisser jouer la loi du marché jusqu'à un certain point. Qu'est-ce que le marché, si ce n'est la loi de la jungle, de la nature, alors que la civilisation, c'est la lutte contre la nature.

Si on enlève la virgule, $J C P$ est intégré dans la proposition, i. e. rendu endophrastique, et active seulement son sens «affirmatif». Il ne permet plus l'enchaînement argumentatif d'opposition «loi du marché» / «civilisation». De même, dans l'exemple suivant où $J C P$ est endophrastique (prédicat de mouvement contraignant), une séparation est impossible si l'on souhaite préserver le bon fonctionnement de l'enchaînement:

[39] En calquant le titre d'un célèbre essai de Sartre, abondamment cité, Eribon le suit jusqu'à un certain point. De même qu'il rejoint Hannah Arendt [...].

?? En calquant le titre d'un célèbre essai de Sartre, abondamment cité, Eribon le suit, jusqu'à un certain point. De même qu'il rejoint Hannah Arendt [...].

* En calquant le titre d'un célèbre essai de Sartre, abondamment cité, Eribon le suit. Jusqu'à un certain point. De même qu'il rejoint Hannah Arendt [...].

(M)

Lorsque $J C P$ est indépendant, sa présence est nécessaire pour faire fonctionner l'enchaînement logique, contrairement à l'emploi intégré. Sous cette forme, JCP a un fonctionnement proche de celui de mais, comme opérateur argumentatif au niveau du contenu asserté, par exemple:

[40] Un hiatus que l'on aime. Jusqu'à un certain point. L'esthétique du pire a ses limites. * Un hiatus que l'on aime. ø L'esthétique du pire a ses limites.

Un hiatus que l'on aime. Mais l'esthétique du pire a ses limites.

(M) 
Cet usage de $J C P$ correspond alors à un «Oui, mais... », i. e. une affirmation bivalente qui a) accepte les conclusions du discours précédent et $b$ ) introduit parallèlement une orientation argumentative opposée.

\subsection{La structure argumentative de $J C P$}

Nous préciserons maintenant les effets structurants du sens «Oui, mais... » de $J C P$ indépendant. Soit l'exemple suivant:

[4I] Les États-Unis estiment que la Corée du Nord est plus vulnérable que jamais, ce qui est exact... jusqu'à un certain point. Certes, le pays dépend largement de l'aide extérieure pour nourrir sa population, dont les conditions de vie ont été aggravées par l'hiver et des réformes économiques qui ont entrâné une flambée des prix. Mais la Corée du Nord reste un «État-secte» galvanisé par un nationalisme farouche qui, depuis un demi-siècle, cultive une «mentalité d'assiégé».

(M)

Dans un tel cas de figure, JCP coorganise l'argumentation concessive. Dans l'exemple choisi, cette structuration est très explicite. Elle se fait conjointement avec les opérateurs certes et mais. Certes et mais fonctionnent d'une manière qui peut être schématisée comme suit (d'après Maingueneau, $1997: 58)^{18}$ :

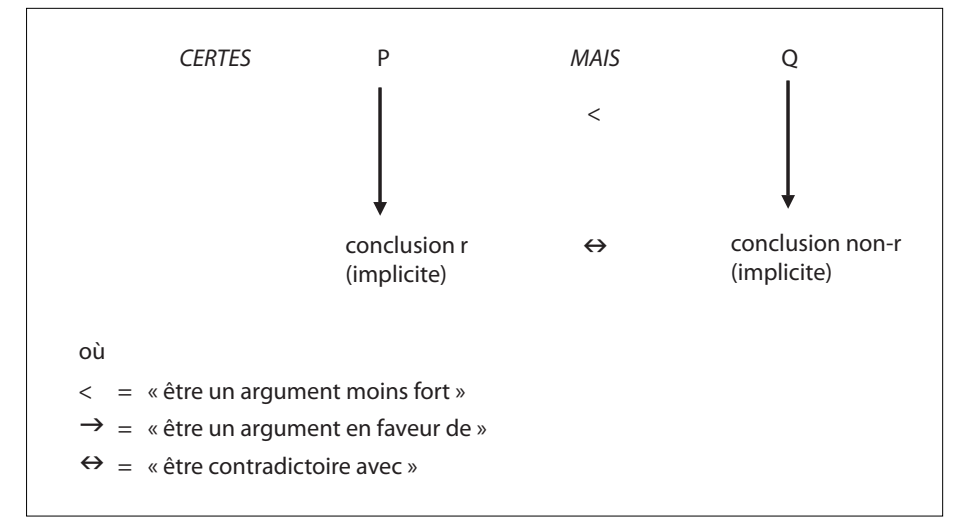

Schéma 2. Structure argumentative d'opposition certes... mais...

Dans notre exemple:

$\mathrm{P}=$ la Corée du Nord dépend de l'aide extérieure

$\mathrm{r}=$ la Corée du Nord est plus vulnérable que jamais

$\mathrm{Q}=$ la Corée du Nord reste un «État-secte»

non- $r$ = la Corée du Nord n'est pas aussi vulnérable qu'on le pense

18. Cette présentation et l'analyse polyphonique (2.4) que nous empruntons à Maingueneau ont été proposées par Ducrot (1984, chap. VIII). 
JCP annonce ces deux volets argumentatifs $\mathrm{P}$ et $\mathrm{Q}$, en parfait accord avec sa double valeur, affirmative d'un côté et négative de l'autre. $\mathrm{P}$ correspond au «Oui», $\mathrm{Q}$ $\mathrm{au}$ «mais» dans JCP. En employant des termes de Roulet et al. (I99I), JCP constitue un acte directeur d'argumentation qui est appuyé par deux arguments subordonnés. L’un, $\mathrm{P}$, est concédé pour mieux faire valoir $\mathrm{Q}$.

Si on supprime tous les opérateurs argumentatifs de l'exemple, il semble d'abord que mais est essentiel pour son fonctionnement.

[42] Les États-Unis estiment que la Corée du Nord est plus vulnérable que jamais, ce qui est exact... (ø). (ø) Le pays dépend largement de l'aide extérieure pour nourrir sa population, dont les conditions de vie ont été aggravées par l'hiver et des réformes économiques qui ont entraîné une flambée des prix. $\left.{ }^{*} \varnothing\right)$ La Corée du Nord reste un «État-secte» galvanisé par un nationalisme farouche qui, depuis un demi-siècle, cultive une «mentalité d'assiégé».

$J C P$ et certes, en revanche, peuvent être supprimés. L'argumentation en faveur de $\mathrm{P}$ est toujours récupérable. En l'absence de JCP et de certes, il faut tout simplement davantage de calcul pour saisir les inférences implicites de l'enchaînement «Mais [...]»:

[43] Les États-Unis estiment que la Corée du Nord est plus vulnérable que jamais, ce qui est exact... (ø). (ø) Le pays dépend largement de l'aide extérieure pour nourrir sa population, dont les conditions de vie ont été aggravées par l'hiver et des réformes économiques qui ont entraîné une flambée des prix. Mais la Corée du Nord reste un «État-secte» galvanisé par un nationalisme farouche qui, depuis un demi siècle, cultive une «mentalité d'assiégé».

La logique du paragraphe «fonctionne» toujours. À cette différence que le lecteur ne découvre qu'a posteriori, à partir de l'instruction procédurale donnée par mais, l'orientation argumentative imposée : il faut «[donner] préférence, dans le contexte discursif où se trouve l'ensemble $\mathrm{P}$ mais $\mathrm{Q}$, aux possibilités argumentatives liées au second segment» (Ducrot, 1993: 49). JCP et certes font alors un travail de préparation du calcul des inférences et rendent l'intention argumentative, et la structure qui la sous-tend, plus accessible, fonction rhétorique qui est confirmée généralement pour les connecteurs (cf. Mauranen, I993: I62 sq.). Est-ce que les deux adverbiaux sont nécessaires pour cela? Non, JCP seul suffit:

[44] Les États-Unis estiment que la Corée du Nord est plus vulnérable que jamais, ce qui est exact... jusqu’à un certain point. (ø) Le pays dépend largement de l'aide extérieure pour nourrir sa population, dont les conditions de vie ont été aggravées par l'hiver et des réformes économiques qui ont entraîné une flambée des prix. Mais la Corée du Nord reste un «État-secte» galvanisé par un nationalisme farouche qui, depuis un demi-siècle, cultive une «mentalité d'assiégé».

Là réside toute la force de JCP. Il contient en quelque sorte le «certes» (de «certes $\mathrm{P}$ mais $\mathrm{Q}$ ), et $\mathrm{P}$ peut alors être introduit directement, comme dans [44], 
avant «mais Q». Qu'en est-il de l'indépendance de $J C P$ par rapport à l'opérateur mais? La locution à elle seule, peut-elle activer le schéma argumentatif esquissé ci-dessus? On trouve, en effet, des emplois argumentatifs de $J C P$ où seul le lexique et les inférences contextuelles (via les connaissances du monde) suffisent, associés à l'orientation argumentative de l'adverbial, pour faire fonctionner la structure concessive. Par exemple:

[45] Tous cependant, n'ont pas tiré un trait sur la diversification. «Avoir plusieurs métiers permet de lisser les effets de Cycle et de diminuer la prime de risque exigée par vos banquiers et vos actionnaires», explique Philippe Camus [...]. Les marchés boursiers admettent l'argument, jusqu'à un certain point. Pour eux, le risque est grand d'utiliser les capacités financières générées par des activités rentables pour financer des métiers qui le sont beaucoup moins. Les diversifications hasardeuses de la Générale des eaux ou de la Lyonnaise des eaux dans l'immobilier, le câble ou la santé, financées par les cash-flows confortables provenant des activités liées à la distribution, ont servi de leçon aux actionnaires. Ils préfèrent dorénavant investir dans les filiales cotées aux contours bien définis plutôt que dans leur maison mère.

(M)

Dans cet extrait, $J C P$ oriente l'argumentation vers la conclusion que les marchés boursiers n'ont pas confiance en la diversification des activités des grands groupes. L'assertion «admettent l'argument» est affaiblie par la locution. Les conclusions à en tirer sont ainsi présentées comme plus faibles que celles générées par la suite du discours. Schématiquement:
P: l'argument est valable
conclusion: on peut s'intéresser à des groupes qui diversifient leurs activités
Q: il existe plusieurs cas de diversifications hasardeuses qui servent de leçon
conclusion: la diversification est un risque

La conclusion de cette argumentation sert de clôture du paragraphe : les actionnaires préfèrent des sociétés dont les activités ont des bornes claires. Sans $J C P$, le discours ne fonctionne plus:

[46] ?? Tous cependant, n'ont pas tiré un trait sur la diversification. «Avoir plusieurs métiers permet de lisser les effets de Cycle et de diminuer la prime de risque exigée par vos banquiers et vos actionnaires», explique Philippe Camus [...]. Les marchés boursiers admettent l'argument, (ø). Pour eux, le risque est grand d'utiliser les capacités financières générées par des activités rentables pour financer des métiers qui le sont beaucoup moins. Les diversifications hasardeuses de la Générale des eaux ou de la Lyonnaise des eaux dans l'immobilier, le câble ou la santé, financées par les cash-flows confortables provenant des activités liées à la distribution, ont servi de leçon aux actionnaires. Ils préfèrent dorénavant investir dans les filiales cotées aux contours bien définis plutôt que dans leur maison mère. 
Souvent, le mouvement argumentatif à partir du pivot $J C P$ enchaîne directement avec la contre-argumentation $\mathrm{Q}$. Dans ces cas, $\mathrm{Q}$ est une négation sous-entendue de $P$ et les raisons de cette contre-conclusion sont ce qui suit. Les arguments pour Q sont introduits par car ou par les deux-points, voir par exemple:

[47] Elle lui reproche une faute politique - faire des «concessions à l'orthodoxie de gauche» - et une erreur d'analyse - se croire seul «légitimé à porter les intérêts des travailleurs». Jusqu’à un certain point... Car M. Jospin a dévoilé une autre facette de sa pensée en affirmant, en septembre 1999, que «les salariés existent, les syndicats aussi», et qu'«il ne faut pas tout attendre de l'État et du gouvernement».

(M)

[48] La municipalité semble avoir compris que l'environnement est l'unique richesse de Nakamura. Jusqu'à un certain point: car elle est aussi en train d'abattre des collines pour regagner des terrains sur la mer.

(M)

La négation restrictive en ne... que jusqu'à un certain point ou sous la forme jusqu'à un certain point seulement a le même effet structurant. Cet effet semble en accord avec une conceptualisation de la négation selon laquelle celle-ci implique une polémique de points de vue opposés (cf. par exemple Ducrot et al., 1980: 50 sq.; Ducrot et Schaeffer, 1995: 700 sq. et 545 sq.), une polyphonie ${ }^{19}$ coordonnant plusieurs énoncés. Dans cette perspective, $J C P$ est le commentaire restrictif porté sur l'affirmative et suivi des arguments qui infirment $\mathrm{P}$, l'assertion. Par exemple:

[49] Sans Hiroshima, le Japon aurait dû déraciner le totalitarisme comme l'Allemagne le fit avec le nazisme. Ce ne fut le cas que jusqu'à un certain point: avant d'avoir été oppresseurs, les Japonais se sentent le premier peuple atomisé.

[5o] Le chef de la guérilla a, semble-t-il, mordu à l'hameçon. Mais jusqu'à un certain point seulement, puisqu'à l'occasion de la fête pour le septième anniversaire de l'EZLN, à laquelle il n'a pas participé, le «commandant David», l'un de ses plus proches lieutenants, a demandé à Vicente Fox d'aller encore plus loin et de fermer toutes les bases militaires - sept au total - installées au Chiapas.

(M)

19. Ducrot résume l'analyse polyphonique de la négation ainsi: «Je proposerai de décrire tout énoncé de la forme non-p comme accomplissement de deux actes illocutionnaires: l'un est l'affirmation de $p$ par un énonciateur E s'adressant à un destinataire $\mathrm{D}$, l'autre est le rejet de cette affirmation, rejet attribué à un énonciateur $\mathrm{E}_{2}$ s'adressant à $\mathrm{D}_{2}$. [...] $\mathrm{E}_{2}$ (celui qui rejette) est en règle générale identifié avec le locuteur; [...] $\mathrm{D}_{2}$ (le destinataire du refus) est en règle générale identifié avec l'allocutaire» (Ducrot, I980: 49 sq.). 
Un emploi particulier et fréquent est constitué par des phrases averbales ${ }^{20}$. Les variantes suivantes issues de notre corpus sont synonymes et initient le même schéma argumentatif:

Jusqu’à un certain point.

= Jusqu'à un certain point seulement.

= Mais jusqu'à un certain point.

= Mais jusqu'à un certain point seulement.

La première version est la plus réduite, la dernière la variante la plus explicite de l'orientation argumentative conférée. On peut facilement les échanger l'une avec l'autre ('original est marqué un gras):

[51] Le spectacle du chorégraphe Marco Berrettini Je m'appelle Emil Sturmwetter... et moi, c'est Maryvonne von Strudelberg, vu sous le Tipi de Beaubourg, agace autant qu'il fascine. Un hiatus que l'on aime. [Jusqu’à un certain point / Jusqu'à un certain point seulement / Mais jusqu'à un certain point / Mais jusqu'à un certain point seulement]. L'esthétique du pire a ses limites.

(M)

[52] Comme les autres conglomérats (zaibatsu), Mitsubishi fut touché par les mesures de décartellisation imposées par les Américains en 1947. [Jusqu’à un certain point/Jusqu’à un certain point seulement / Mais jusqu'à un certain point / Mais jusqu'à un certain point seulement]. Au moment de la défaite, Mitsubishi possédait pratiquement tout le quartier des affaires de Tokyo: Marunouchi.

(M)

[53] À l'heure de la globalisation, les idéogrammes peuvent constituer un ferment de synergie régionale. C'est la raison principale pour laquelle Séoul veut les réintroduire. Mais l'informatique a un effet ambivalent sur leur utilisation. L'ordinateur facilite leur écriture et donc leur pratique - [jusqu'à un certain point / jusqu'à un certain point seulement / mais jusqu'à un certain point / mais jusqu'à un certain point seulement]. Car, en même temps, se perd le «travail de la main» dans leur apprentissage.

(M)

[54] Curieusement, ce film ressemble à un téléfilm [...]. Le talent de Fabrice Luchini est de parvenir à rendre crédible ce scénario bizarre. [Jusqu’à un certain point / Jusqu’à un certain point seulement / Mais jusqu'à un certain point / Mais jusqu’à un certain point seulement]. L'arrivée finale des gendarmes était, bien que la soirée ne soit pas si avancée que cela, une véritable bénédiction. Une douce torpeur envahissait le chroniqueur. C'était peut-être l'effet de toutes ces anesthésies.

(M)

20. Lorsque «Jusqu'à un certain point» est réponse à une question totale, l'effet d'inversion argumentative peut être suspendu car JCP présente la simple assertion (cf. I.3, [27], cf. [74]). 
Cet usage souligne de manière marquée le potentiel argumentatif de JCP. L'adverbial est utilisé avec insistance grâce à une ponctuation forte qui l'isole et qui en révèle tout l'apport polyphonique. Le procédé est donc rhétoriquement très efficace et fait de $J C P$ un outil (pivot) puissant dans la structuration de l'argumentation.

Tandis que les deux variantes de négation restrictive rejoignent les constructions avec $J C P$ syntaxiquement isolé, l'emploi argumentatif esquissé ci-dessus n'est pas possible avec $J C P$ modal détaché en tête de phrase. $J C P$ n'y fonctionne évidemment pas comme une affirmation indépendante mais marque la suspension de l'assertion qui suit. Comme pour l'emploi endophrastique de $J C P$ postverbal non détaché, l'enchaînement devrait continuer dans l'orientation de la proposition modifiée, i. e. dans le sens affirmatif. Ainsi dans l'exemple suivant, différent de [4I], le volet introduit par mais ne peut se lire comme subordonné à $J C P$. Il doit être interprété comme un nouveau segment de discours:

[55] \# Jusqu'à un certain point, la Corée du Nord est plus vulnérable que jamais. Le pays dépend largement de l'aide extérieure pour nourrir sa population, dont les conditions de vie ont été aggravées par l'hiver et des réformes économiques qui ont entraîné une flambée des prix. Mais la Corée du Nord reste un «État-secte» galvanisé par un nationalisme farouche qui, depuis un demi-siècle, cultive une «mentalité d'assiégé».

En résumé, la structure argumentative avec $J C P$ se construit ainsi:

- O JCP

- CERTES $\mathrm{P}=$ segment orientant vers les mêmes conclusions à tirer de $\mathrm{O}$

- MAIS $Q=$ segment orientant vers des conclusions opposées à $\mathrm{O}$, à tirer de $\mathrm{Q}$, présentées comme préférables à celles de $\mathrm{P}$

Nous allons maintenant étudier l'effet polyphonique des structures argumentatives en $J C P$ et nous achèverons cette partie par une comparaison de nos résultats avec le modèle des modificateurs déréalisants de Ducrot (1995, 1998).

\section{4. $J C P$ et l'effet de polyphonie}

L'emploi argumentatif de $J C P$ est étroitement lié à des phénomènes de polyphonie. Nous avons vu que $J C P$ active un volet argumentatif «certes» et un contre-volet «mais», même s'ils peuvent rester virtuels. Nous résumons avec Maingueneau (1997: 59) ce qui se passe dans un système où certes et mais interagissent, comme dans l'exemple $[4 \mathrm{I}]$ :

En effet, quand un locuteur déclare «certes $\mathrm{P}$ mais $\mathrm{Q}$ » il attribue $\mathrm{P}$ à un objecteur qu'il met en scène dans son discours et dont il rejette le point de vue au profit de $\mathrm{Q}$. On assiste donc à l'affrontement entre deux points de vue successifs argumentant dans des directions opposées. Le locuteur se dit d'accord avec celui qui soutient $\mathrm{P}$ mais s'en distancie. Dans ce mouvement il assimile son allocutaire à cet «énonciateur» de $\mathrm{P}$, à qui il fait une concession. 
Cet effet de polyphonie est accentué s'il y a une ponctuation forte qui sépare les opérateurs des propositions. Ce procédé imite un tour de parole entre locuteur et interlocuteur tel qu'on le trouve dans une suite question-réponse. Il y a plusieurs

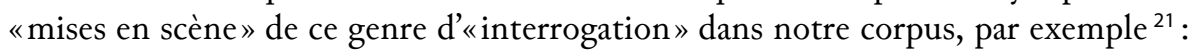

[56] Finie l'ère des banlieues et des grandes migrations pendulaires? Jusqu'à un certain point. Les appartements des tours du quartier d'Azabu sont conçus pour les étrangers ou des Japonais fortunés.

(M)

Mais on le trouve aussi sous forme d'assertions:

[57] Avec ses feuilles persistantes en hiver, le houx semble être un symbole parfait de la résistance au froid. Jusqu'à un certain point. Car c'est précisément le froid hivernal qui limite son extension vers le nord.

(M)

Dans les deux cas, la première proposition, modifiée par $J C P$, est attribuée à un interlocuteur fictif. Cet interlocuteur renvoie au lecteur lui-même. JCP impose un jeu rhétorique par lequel le lecteur doit s'identifier avec une position pour être mieux «instruit» du contraire. Dans le dernier exemple, on est contraint à faire comme si on admettait l'adéquation symbolique du houx, seulement pour que le locuteur nous dise implicitement «Ce n'est pas exact» et puisse enchaîner avec «Car $[. .$.$] ».$

\subsection{JCP et le modèle des modificateurs déréalisants de Ducrot $(1995,1998)$}

Le fonctionnement de JCP que nous venons d'exposer est compatible avec un modèle proposé par Ducrot (1995, 1998) ${ }^{22}$ dans la suite de la «Théorie de l'argumentation dans la langue», qui introduit les notions de modificateur réalisant et modificateur déréalisant: «Un mot Y est dit "modificateur déréalisant" (MD) par rapport à un mot $\mathrm{X}$ si le syntagme $\mathrm{XY}$ a une orientation inverse ou une force argumentative inférieure à celle de X. Dans le cadre de la "Théorie de l'argumentation dans la langue", on dit que $\mathrm{Y}$ s'oppose à l'application des formes topiques intrinsèques à $\mathrm{X}$. Un critère pour reconnaître un MD est l'impossibilité de dire, sauf intention argumentative complexe, "X et même XY"» (I995: I). Un mot est modificateur réalisant (MR) d'un prédicat s'il renforce l'application des topoï liés à ce prédicat. Il ne peut alors y avoir d'enchaînement «X mais $\mathrm{XY}$ ».

La notion de MD/MR est d'abord appliquée à des éléments lexicaux comme des adjectifs ou des adverbes, par exemple ${ }^{23}$ :

21. L'exemple n'est pas un vrai échange entre deux locuteurs.

22. C'est V. Lenepveu qui nous a suggéré la proximité de notre analyse avec les conclusions de O. Ducrot et son modèle de modificateurs.

23. Nous reprenons la notation de Ducrot: @ marque «la possibilité d'une [...] opposition immédiate entre $\mathrm{X}$ et $\mathrm{XY}$, indépendante de toute conclusion précise. Si, au contraire, il est nécessaire d'imaginer, pour 
[58a] Il y a une solution, mais @ difficile / \# facile.

[58b] Il y a un problème, mais \# difficile / @ facile.

[58c] Il y a une solution, et même @ facile / \# difficile.

[58d] Il y a un problème, et même \# facile / @ difficile.

Aussi, on doit distinguer entre MD atténuateurs et MD inverseurs pour expliquer la différence d'orientation argumentative des exemples suivants, empruntés à Ducrot (I995), donnant un argument suivi de sa conclusion :

[59] Le changement de Paris est lent; tu ne seras pas dépaysé.

[6o] Il y a eu de lents changements à Paris; tu seras peut-être dépaysé.

Un topos supposé œuvrer dans l'argument veut que des changements dans une ville familière puissent dépayser une personne. Dans [59], la conclusion est inverse à la conclusion qu'on peut tirer de l'existence d'un changement. Ce fait est réalisé par la présence de «lent», MD inverseur. Le même adjectif, en position d'épithète en [6o], ne modifie pas l'orientation générale de l'argument, il l'affaiblit seulement. «Lent»y est alors MD atténuateur.

Ducrot fait l'hypothèse que «les MD deviennent inverseurs lorsque, et seulement lorsque le locuteur en fait le propos de son énonciation» (1995: 153). Dans sa terminologie, l'exemple [59] présente une affirmation présupposée: «il y a eu du changement»; et une prédication qui pose que «ce changement était lent» et qui constitue le propos de l'énonciation. L'exemple [6o], au contraire, pose une seule prédication : «y avoir de lents changements». Un MD atténuateur s'oppose aux topoï du prédicat sans pouvoir les annuler, car l'élément qui fait l'objet de l'énonciation est le prédicat et l'orientation argumentative est celle qui en découle. Par exemple, dans [6o], emprunté à Ducrot (I995), l'objet de l'énonciation est le changement, son orientation argumentative le «dépaysement». L'épithète «lent» ne peut qu'atténuer, sans inverser cette conclusion, car il constitue une prédication inférieure à celle de «y avoir du changement». C'est pourquoi Ducrot a choisi d'insérer un «peut-être» dans la conclusion : il exprimer l'atténuation du propos tout en maintenant la conclusion «dépaysement». Enfin, les MR restent immuns à la différence entre une seule ou deux affirmations, présupposée et posée, comprises en un énoncé, car ils orientent toujours dans le sens des topoï du prédicat.

Ducrot étend l'approche en termes de modificateurs, aux adverbiaux quantitatifs, plus précisément au couple de quantifieurs peu/un peu. Antérieurement (Ducrot, I980), il les avait opposés, «en insistant sur le fait que peu inverse l'orientation

comprendre cet enchaînement, une argumentation en faveur d'une troisième proposition, j'affecterai l'enchaînement du symbole \#» (I995: I48). 
argumentative du syntagme où il est introduit, alors que un peu la conserve, tout en l'atténuant: le même type de conclusion est autorisé par "Pierre a mangé" et "Pierre a un peu mangé", contraire à ce qu'on conclurait de "Pierre a peu mangé” (Ducrot, 1995: 153). Peu est ainsi proche de la négation, un peu de l'assertion. Ducrot (1995, 1998) réanalyse les deux, peu et également un peu, comme MD : peu comme MD inverseur, un peu comme MD atténuateur, pour rendre compte de contre-exemples tel le suivant, qui semble ordonner un peu et peu sur une même échelle:

[61] Quoiqu'il doive retomber [dans le péché] un peu après et même peu après.

(cf. Ducrot, 1998: 366 sq.)

Suivant le postulat que, pour être inverseur, un MD doit intégrer un énoncé qui à la fois présuppose et pose quelque chose, Ducrot (1995; cf. l'analyse de Ducrot et al., 1980: 194 sq.) dit que un peu pose une affirmation tandis que peu la présuppose et y applique une restriction (cf. Ducrot, 1995: 154, Parle un peu! vs Parle peu.). Il propose de concevoir un même morphème abstrait, CHOUIA, dont peu et un peu sont des occurrences.

$J C P$ aussi peut s'analyser comme un MD atténuateur et inverseur, selon le cas, et comme une occurrence particulière de la notion CHOUIA. Le test en mais montre la nature constante de $\mathrm{MD}$ de $J C P$ qui est toujours incompatible avec l'augmentatif même:

[62a] @ Il l'aime, mais jusqu'à un certain point.

[62b] ?? Il l'aime, et même jusqu'à un certain point.

[62c] @ Il le hait, mais jusqu’à un certain point.

[62d] ?? Il le hait, et même jusqu’à un certain point.

[62e] @ C'est une figure emblématique, mais jusqu’à un certain point.

[62f] ?? C'est une figure emblématique, et même jusqu'à un certain point

Nous dirions que pour $J C P$, la différence entre $\mathrm{MD}$ atténuateur et $\mathrm{MD}$ inverseur tient à ce que $J C P$ constitue ou non une affirmation indépendante portant sur une prédication déjà posée (cf. nos explications sous 2.2). Deux cas de MD atténuateur peuvent être répertoriés. Le premier est évident. Il se présente quand $J C P$ est endophrastique, intra-prédicatif et apparait dans un énoncé assertif. Le discours suivant est alors orienté dans le sens des conclusions qu'on peut tirer de l'affirmation, par exemple:

[63] Cela a fonctionné jusqu'à un certain point. Un des signes en a été l'apparition de la fameuse oligarchie qui a profité de cette nouvelle économie de marché pour s'enrichir [...].

(M) 
Dans cet extrait, l'argumentation continue dans le sens «avoir fonctionné». Dans notre corpus, cet emploi est rare, mais toutes les occurrences de ce type confirment une telle continuation de l'orientation donnée par l'affirmation. En revanche, si $J C P$ est sous la portée d'une forme de restriction (ne... que..., seulement), l'effet est inverse, et l'argumentation est orientée dans le sens opposé des conclusions à tirer de l'affirmation:

[64] Les atouts dont ils se targuent - la spécialisation des consultants par secteur d'activité, la formation continue dispensée aux plus jeunes - ne leur permettent de continuer à faire du sur-mesure que jusqu'à un certain point. La profession «aurait en réalité besoin de moyens supplémentaires», insiste Francis Rousseau [...].

(M)

L'analyse polyphonique de Ducrot permet d'expliquer cet effet très simplement, par le fait qu'une restriction constitue une prédication indépendante. JCP est donc en mesure d'inverser parce qu'il fait partie d'un acte restrictif supérieur à l'assertion, remplissant ainsi la condition des MD inverseurs d'être «le propos de [1]a parole» de l'énonciateur (Ducrot, 1995: 153). C'est pourquoi un emploi endophrastique peut seulement être inverseur si le prédicat est repris comme un présupposé sur lequel un commentaire peut ensuite être introduit par une deuxième prédication, voir ainsi :

[65] Ces expérimentations étaient jusqu'à un certain point secrètes.

vs

[66] Secrètes, ces expérimentations l'étaient jusqu'à un certain point: c'est la kanpeitai, police militaire, qui alimentait celle-ci en cobayes humains; en outre, l'Unité employait des médecins civils, et elle était en étroites relations avec des hôpitaux au Japon, auxquels étaient communiquées les conclusions des expériences. «Le monde médecin japonais savait parfaitement comment celles-ci étaient obtenues», estime Keiichi Tsuneishi, historien des sciences et expert de l'histoire de l'Unité 73I.

(M)

Un deuxième cas présentant $J C P$ comme $\mathrm{MD}$ atténuateur est l'emploi exophrastique détaché en tête de phrase ${ }^{24}$ :

[67] Jusqu'à un certain point, on peut dire que l'affaiblissement du dollar est calculé: tout pays qui cherche à sortir de la récession va essayer d'affaiblir sa monnaie, et il le fait dans son intérêt national. [...] Les États-Unis n'ont pu se permettre d'avoir de tels déficits que parce que le dollar était l'unique monnaie dominante.

(M)

24. Nous ne commentons pas, dans la présente étude, les emplois à l'intérieur d'une phrase, car ils présentent une grande complexité d'analyse, chaque cas pouvant varier en fonction du cotexte particulier dans lequel il apparaît et de l’orientation argumentative générale donnée au texte dans lequel il figure. 
En effet, même si l'argumentation peut être réorientée par la suite grâce à l'introduction d'un nouvel opérateur argumentatif fort comme mais ou cependant, dans cette position, $J C P$ maintient l'affirmation qu'il modifie. Un MD comme JCP ne peut «inverser» que s'il y a une affirmation dont il peut inverser l'orientation. En tête de phrase, JCP modal suspend toute affirmation.

Finalement, l'emploi MD inverseur est celui que nous avons décrit sous 2.2 comme «indépendant», i. e. lorsque $J C P$ est en position finale et constitue à lui seul une affirmation complète, un commentaire, ce qui se reflète dans un détachement fort par la ponctuation (virgule, point, points de suspension, introduction par mais). Voici un exemple permettant de rappeler cet emploi:

[68] On pourrait prolonger à l'infini ce parallèle. Il est éloquent. Mais jusqu’à un certain point. Les Francs n'avaient aucun «droit historique» sur la Terre sainte.

$J C P$ inverse alors l'orientation argumentative de l'affirmation pour introduire des contre-arguments, annulant la validité de «ce parallèle éloquent».

Nous n'avons pas pu aborder toutes les questions que l'approche en termes de MD/MR peut laisser ouvertes, par exemple la relation entre quantification et effet de modificateur, ou le statut syntaxique différent d'expressions quantifiantes comme un peu/peu et d'adverbiaux polyfonctionnels comme JCP. Des croisements supplémentaires d'approches permettront certainement de clarifier ces points et de compléter l'analyse. Il nous reste maintenant à présenter les structures argumentatives trouvées dans notre corpus.

\section{Analyse du corpus: les types de structure argumentative associés à l'emploi de $J C P$}

\subsection{Particularités du corpus}

Le journal Le Monde de 1980 à 2006 sur CD-ROM contient 278 occurrences de $J C P$. Ce faible chiffre fait écho aux résultats maigres obtenus lors d'une recherche sur Frantext pour une période contemporaine (1900-2006) où le total est de 213 occurrences ${ }^{25}$. La locution ne semble donc pas être si fréquente, mais une comparaison avec son emploi à l'oral reste à faire.

Il faut remarquer que bon nombre des textes comprenant une occurrence de $J C P$, sans être majoritaires, ne sont pas des textes journalistiques au sens strict. Ce sont souvent des critiques de film, théâtre ou littérature, des dossiers culturels ou philosophiques voire des interviews. On ne semble donc pas être dans le genre «texte d'information» pur, mais face à des types d'écrit journalistique qui permettent plus

25. Après élimination des doublons et des occurrences où JCP ne représente pas la locution figée dont on traite ici. 
facilement l'affichage ouvert d'une part de subjectivité... fait qui ne surprend pas vu la charge modale, synonyme de subjectivité, que porte $J C P$.

Aussi, l'emploi de $J C P$ dans le corpus analysé semble en quelque sorte être une idiosyncrasie stylistique d'un journaliste particulier: 85 occurrences, i. e. $3 \mathrm{I} \% \mathrm{des}$ occurrences de JCP, se trouvent dans des textes signés Philippe Pons.

Stylistiquement, il y a quelques particularités syntaxiques de l'usage de $J C P$ dans les textes du Monde. Dans ce corpus, on trouve JCP intégré dans des phrases ${ }^{26}$ averbales, comme dans:

[69] Jusqu’à un certain point, certainement.

[70] C'est quand même encore une guerre d'école. Jusqu'à un certain point. (M)

Frantext ne donne aucune occurrence de ce type. Il y a également plusieurs enchaînements du type question/réponse où $J C P$ apparaît dans la partie réponse. Dans Frantext, ces enchaînements sont réduits à une seule occurrence.

Dans seulement $\mathrm{I} 8$ phrases, i. e. $6,5 \%$ des occurrences, JCP prend la position en tête de phrase. C'est en position finale qu'il prend nettement tout son pouvoir argumentatif. Dans un bon tiers des cas (95 occurrences, i. e. 34,2 \%), JCP est le dernier élément d'une phrase.

\subsection{Les structures discursives potentielles}

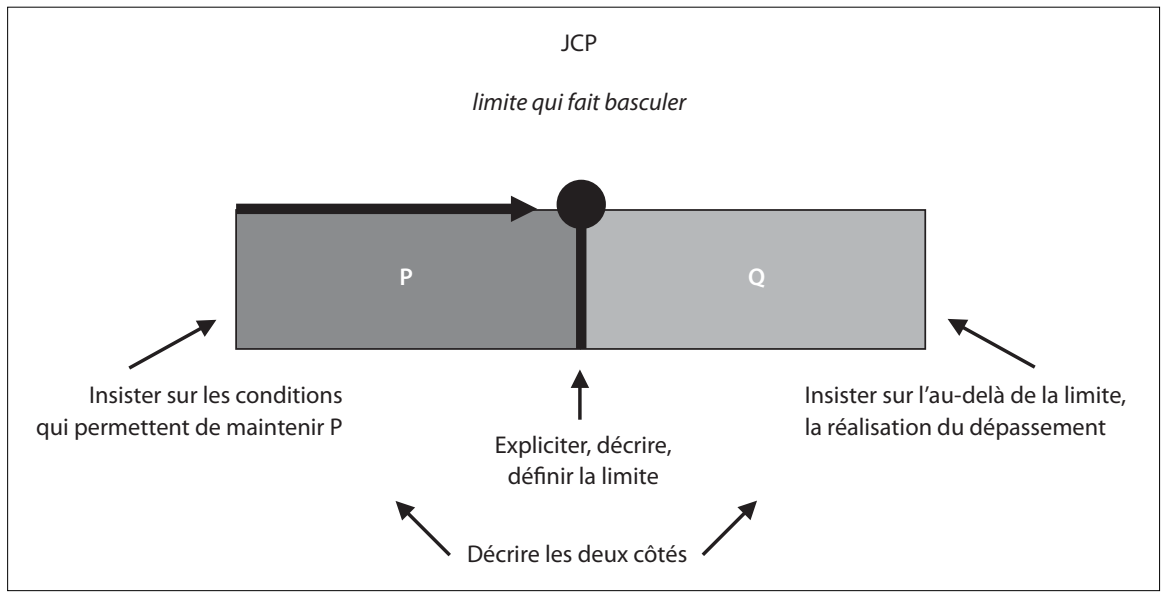

Schéma 3. Les champs d'argumentation possibles

26. Nous utilisons la notion de «phrase» de manière très réduite, c'est-à-dire correspondant à une suite de mots formant une proposition qui est délimitée par la ponctuation forte du point. 
Nous avons donné sous 2 le type général de structuration que $J C P$ peut introduire. Comme nous allons le voir, ce n'est pas la seule structure trouvée dans notre corpus d'occurrences. Théoriquement, suivant la métaphore du parcours borné, JCP devrait permettre les organisations discursives indiquées dans le schéma 2. Nous avons effectivement trouvé des instances de chaque possibilité. Mais la structure décrite dans les paragraphes précédents, correspondant à «insister sur l'au-delà», est tout de même la plus exploitée (voir schéma 3).

\subsection{Les structures argumentatives avec $J C P$ recensées dans Le Monde}

Les exemples suivants illustrent les structures argumentatives avec $J C P$ en position finale. Comme annoncé (cf. le schéma 2), on trouve quelques exemples où la présence de $J C P$ ne déclenche pas une suite discursive qui fait s'opposer deux orientations argumentatives. Nous résumerons chaque type de structure schématiquement, en employant la notation suivante:

O: segment de propositions ${ }^{27}$

$\rightarrow$ conclusion $\mathrm{r}$ (en faveur des implications de $\mathrm{O}$ )

$\mathrm{P}$ : segment de propositions

$\rightarrow$ conclusion $\mathrm{r}$ (en faveur des implications de $\mathrm{O}$ )

Q: segment de propositions

$\rightarrow$ conclusion non-r (contre la conclusion $r$ et en faveur des implications de $Q$ )

S: nouvelle proposition d'un sujet autre que celui de $\mathrm{O}, \mathrm{P}$ et $\mathrm{Q}$

Nous indiquerons la suite des segments de propositions possibles $(\mathrm{O}, \mathrm{P}, \mathrm{Q}, \mathrm{S})$ et la place de JCP. L'orientation argumentative finale est marquée $\operatorname{par} \Rightarrow$.

Les structures trouvées sont:

I) $\mathrm{O} \rightarrow \mathrm{r} / \mathrm{JCP} / \mathrm{S} \Rightarrow \varnothing$

Dans ce cas, le potentiel argumentatif structurant de $J C P$ reste inactif. $\mathrm{O}$ et $\mathrm{S}$ ne sont pas deux segments liés d'une structure argumentative. Des occurrences montrent que cela peut se faire avec un emploi plutôt endophrastique ainsi qu'avec un emploi plutôt exophrastique :

27. Nous sommes consciente de l'abus de langue mais avons préféré, pour alléger l'exposé, ne pas délimiter par O, P et Q des segments grammaticaux constitutifs d'un propos circonscrit, et distinguer ce dernier d'éléments de contenu. Plutôt, nous utilisons ces majuscules pour désigner un ensemble de contenus qui peut être considéré comme un bloc thématique d'une ou plusieurs propositions orientées vers une conclusion $\mathrm{r}$ ou une contre-conclusion non-r. La formule O / JCP / Q ne transcrit donc pas une phrase comme «C’est vrai jusqu'à un certain point mais pas toujours.», mais, séparément, la proposition «C’est vrai» $(\mathrm{O})$ et la place de $J C P(\mathrm{JCP})$, qui s'insère linéairement après cette proposition, puis la proposition «pas toujours» $(\mathrm{Q})$. La conclusion $\mathrm{r}$ suit directement $\mathrm{O}: \mathrm{O} \rightarrow \mathrm{r}$. La conclusion finale, notée par $\Rightarrow$, est, dans cet exemple: $\Rightarrow$ non-r. Ainsi: $\mathrm{O} \rightarrow \mathrm{r} / \mathrm{JCP} / \mathrm{Q} \Rightarrow$ non-r. 
[71] La dévotion de son mari, l'amour étouffant et exclusif de ses fils la protègent jusqu'à un certain point. Valeria Golino parvient à faire cohabiter en un seul personnage la déraison la plus effrayante, la vulnérabilité la plus désarmante et la séduction la plus impérieuse.

$(\mathrm{M})$

[72] J'avais trouvé le déménagement à Montréal un peu difficile, surtout après Toronto, où j'ai passé seize ans et où tout le monde vit en harmonie, jusqu'à un certain point. À Montréal, les tensions sont plus vives.

(M)

2) $\mathrm{O} \rightarrow \mathrm{r} / \mathrm{JCP} / \mathrm{P} \Rightarrow \mathrm{r}$

Dans ce type d'exemples, la conclusion $r$ est en accord avec les implications de $\mathrm{O}$ et de P. JCP ne fait pas pivoter l'orientation argumentative:

[73] Cela a fonctionné jusqu'à un certain point. Un des signes en a été l'apparition de la fameuse oligarchie qui a profité de cette nouvelle économie de marché pour s'enrichir [...].

(M)

[74] Tout cela signifie-t-il que la détente est en vue? Jusqu’à un certain point. Avec une économie en faillite, incapable de nourrir sa population, le régime de Pyongyang a besoin de la manne étrangère pour survivre.

(M)

3) $\mathrm{O} \rightarrow \mathrm{r} / \mathrm{JCP} / \mathrm{P} \rightarrow \mathrm{r} / \mathrm{Q} \Rightarrow$ non-r

Comme dans le schéma prototypique, JCP fait pivoter l'argumentation, et le contenu de $\mathrm{Q}$ oriente l'argumentation dans le sens opposé de l'argumentation orientée par $\mathrm{O}$ et $\mathrm{P}$, ainsi dans:

[75] Les États-Unis estiment que la Corée du Nord est plus vulnérable que jamais, ce qui est exact... jusqu'à un certain point. Certes, le pays dépend largement de l'aide extérieure pour nourrir sa population, dont les conditions de vie ont été aggravées par l'hiver et des réformes économiques qui ont entraîné une flambée des prix. Mais la Corée du Nord reste un «État-secte» galvanisé par un nationalisme farouche qui, depuis un demi-siècle, cultive une «mentalité d'assiégé».

(M)

[76] Autour de la table, ils sont une douzaine qui hésitent encore, tandis que des représentants, presque plus nombreux, des collectivités locales et des administrations poitevines s'emploient, si ce n'est à les convaincre, au moins à les rassurer. Jusqu'à un certain point: «Nous sommes prêts à vous aider, annonce Gérard Biette, directeur du développement économique du conseil général de la Vienne. Mais il s'agit de coopération, pas d'assistanat».

(M) 


\section{4) $\mathrm{O} \rightarrow \mathrm{r} / \mathrm{JCP} / \mathrm{Q} \Rightarrow$ non-r}

Cette version est beaucoup plus courante que la précédente. Elle fait l'économie du segment $\mathrm{P}$, qui supporterait une conclusion favorable. On passe tout de suite à $Q$ et ainsi à l'introduction d'une orientation argumentative opposée à $\mathrm{O}$. Le passage de $\mathrm{P}$ à $\mathrm{Q}$ se voit même dédoublé dans le deuxième exemple:

[77] Ils ont aussi appris à respecter les interdictions d'arrosage, jusqu'à un certain point: pourtant, avouons-le, nous ne nous résoudrions qu'en dernière extrémité à ne pas arroser la plante qui le nécessiterait.

(M)

[78] Renault, devenu le premier actionnaire de Nissan, est-il en train d'apprendre aux Japonais les nouvelles règles du jeu du capitalisme? Jusqu'à un certain point. Car les restructurations en cours vont bien au-delà des prises de participation claironnées dans les firmes nippones. Cette entrée massive du capital étranger est nouvelle et constitue un apport d'air frais qui accélère les mutations. C'est le cas chez Nissan, sclérosé et démoralisé. Mais le plan Ghosn n'est que la version radicale de mesures décidées dès 1998. Les entreprises japonaises n'ont pas attendu le débarquement en force du capital étranger pour prendre conscience de la nécessité de changer - au risque de perdre la bataille de la globalisation des marchés.

(M)

\section{5) $\mathrm{O} \rightarrow \mathrm{r} / \mathrm{JCP} / \mathrm{P}+\mathrm{Q} \Rightarrow$ balance entre $\mathrm{r}$ et non-r}

Ce type d'agencement revient dans quelques textes. Il utilise le sens d'un «plus ou moins» de JCP pour mettre en évidence à la fois des éléments en faveur d'une certaine conclusion (r) et des aspects qui pourraient argumenter pour le contraire (non-r). Dans l'exemple qui suit, c'est le marqueur pourvu que et les connaissances du monde qui établissent l'équilibre entre le «pour et le contre» de la démocratisation de Harcourt (les directeurs généraux, les salariés méritants, 3900 francs... ne sont pas ce qu'on dirait du plus «populaire»):

[79] Après avoir failli être racheté par les Japonais il y a quatre ans, Harcourt, le célèbre studio qui, de Simone Signoret à Carole Bouquet, a immortalisé les monstres sacrés, diversifie ses activités et se démocratise... jusqu'à un certain point. Il se diversifie dans la publicité: le persan angora de Whiskas, c'est lui, ainsi que le briquet Zippo et la Safrane-Renault. Et parfois se commet avec les entreprises en tirant le portrait du président-directeur général et des salariés méritants. Côté grand public, pourvu que l'on dispose d'un budget de I 500 francs, il est possible d'obtenir des photos d'identité et, pour 3900 francs, un portrait signé Harcourt.

6) Titre annonçant une bipartition argumentative du texte: $\mathrm{O} / \mathrm{JCP} \Rightarrow$ non-r

$J C P$ peut apparaitre dans des titres ou premières phrases d'un texte, préparant ainsi une bipartition argumentative du texte entier. Celui-ci sera alors structuré 
en des passages qui représentent éventuellement $\mathrm{P}$, nécessairement $\mathrm{Q}$. Deux exemples de titre:

[80] La Formule I adore les caméras. Jusqu’à un certain point.

(M)

[8I] La croissance peut se passer de démocratie... jusqu'à un certain point.

(Internet)

\section{Conclusion}

La présente étude a précisé le caractère hybride de la locution adverbiale jusqu'à un certain point, entre quantifieur et modal, ce qui revient à des emplois où trancher entre portée endophrastique ou exophrastique n'est pas complètement possible. On a vu que des arguments internes associés à la prédication ou à la dimension temporelle de celle-ci, peuvent influer sur l'interprétation de $J C P$. Il est devenu évident que $J C P$ ne peut fonctionner comme cadratif topique (Charolles), étant donné qu'un emploi véridictionnel qui mettrait en relief des valeurs spatio-temporelles, est quasiment introuvable. On a décrit $J C P$ comme space builder dans une perspective cognitive (Fauconnier). Puis l'approche argumentative (Anscombre et Ducrot) s'est révélée très fructueuse pour expliquer le comportement structurant de JCP. JCP est souvent employé comme un opérateur argumentatif comparable, dans son fonctionnement structurant, à mais. Il crée un pont entre trois segments, $\mathrm{O}(+\mathrm{P})$ et $\mathrm{Q}$, qu'il oriente de manière à ce que les conclusions à tirer de $\mathrm{Q}$ soient présentées comme plus pertinentes que celles à tirer de $\mathrm{O}(+\mathrm{P})$. Finalement, nous avons présenté les variantes structurelles cocréées par JCP qu'on peut trouver dans notre corpus du Monde.

Il nous resterait à analyser ce qui se passe avec JCP en position postverbale non finale, pour le comparer avec les résultats obtenus présentement. Nous n'avons pu aborder les effets intonatifs dans ce travail sur corpus écrit. Il est évident que d'importantes informations sur le caractère endo- ou exophrastique d'une occurrence et sur les effets de polyphonie, se jouent à ce niveau à l'oral.

\section{Bibliographie}

Adler, S. et Asnes, M. 2004. Les compléments de degré en jusqu'à. Travaux de linguistique 49 (2) : $133-159$.

Anscombre, J.-C. et Ducrot, O. 1983. L'argumentation dans la langue. Bruxelles: Mardaga.

Caudal, P. et Nicolas, D. 2005. Types of Degrees and Types of Event Structures. Accessible en ligne sur http://d.a.nicolas.free.fr/research/Caudal_Nicolas_Degrees_and_events.pdf.

Charolles, M. I997. L'encadrement du discours - univers, champs, domaines et espaces. Cabiers de recherche linguistique 6: $\mathrm{I}-73$.

Charolles, M. 2003. De la topicalité des adverbiaux détachés en tête de phrase. Travaux de linguistique 47: $\mathrm{II}^{-} \mathrm{S}$. 
Charolles, M. et Vigier, D. 2005. Les adverbiaux en position préverbale: portée cadrative et organisation des discours. Langue française I48: 9-30.

Ducrot, O. 1980. Dire et ne pas dire. Paris: Hermann.

Ducrot, O. 1984. Le dire et le dit. Paris: Éditions de Minuit. Chap. VIII.

Ducrot, O. 1993. Opérateurs argumentatifs et analyse de textes. In W.J. Ashby, M. Mithun et G. Perissinotto (eds), Linguistic Perspectives on the Romance Languages. Amsterdam - Philadelphie: John Benjamins.

Ducrot, O. 1995. Les modificateurs déréalisants. Journal of Pragmatics 24: 145-165.

Ducrot, O. 1998. Quand peu et un peu semblent coorientés. In D. LEEMAN et A. Boone (eds), Du percevoir au dire, hommage à André Joly. Paris: L’Harmattan : 351-373.

Ducrot, O. et al. 1980. Les mots du discours. Paris: Éditions de Minuit.

Ducrot, O. et Schaeffer, J.-M. 1995. Nouveau dictionnaire encyclopédique des sciences du langage. Paris: Seuil.

Fauconnier, G. 1985. Mental Spaces. Cambridge (Mass.) : MIT Press.

Guimier, C. 1996. Les adverbes du français. Le cas des adverbes en -ment. Paris - Gap: Ophrys.

Kleiber, G. et Riegel, M. 1978. Les grammaires floues. In R. Martin (dir.), La notion de recevabilité en linguistique. Paris: Klincksieck: 67-I24.

Lakoff, G. et Johnson, M. 1980. Metaphors We Live by. Chicago: The University of Chicago Press.

LANGACKER, R. 1987. Mouvement abstrait. Langue française 76: 59-76.

LenEPVEu, V. 2009. Intégralement dans le champ des adverbes de complétude. Revue Romane 44 (2): 195-217.

Maingueneau, D. 1997. Pragmatique pour le discours littéraire. Paris: Dunod.

Martin, R. 1983. La logique du sens. Paris: PUF.

Mauranen, A. 1993. Cultural Differences in Academic Rhetoric: a Textlinguistic Study. Francfort - New York: Peter Lang.

Minatsch, W. 2оiо. Les approximateurs quantitatifs entre scalarité et non-scalarité. Langue française 165 : 125 -153.

Molinier, C. et Levrier, F. 2000. Grammaire des adverbes. Description des formes en -ment. Genève - Paris: Droz.

NøjgaARD, M. 1995. Les adverbes français. Copenhague: Munksgaard.

Prince, E.F., Frader, J. et Bosk, C. 1982. On Hedging in Physician-Physician Discourse. In R.J. Di Pietro (ed.), Linguistics and the Professions. Norwood (N.J.) : Ablex.

Rey, A. et Chantreau, S. (dir.) 2007. Dictionnaire des expressions et locutions. Paris: Dictionnaires Le Robert.

Roulet, E. et al. 199I. L'articulation du discours en français contemporain. Berne: Peter Lang.

Schnedecker, C. 2005. Certain et ses avatars (certain $N /$ un certain $N$; certains $N /$ de certains $N$; certains) : approche diachronique. Travaux de Linguistique 50: I3I-I50.

TAlmy, L. 2000. Toward a Cognitive Semantics. Londres: MIT Press. Vol. I et II.

VAn Eemeren, F.H. et al. 1996. Fundamentals of Argumentation Theory. Mahwah (N.J.) : Lawrence Erlbaum. 